Meadow, P. (1956). J. gen. Microbiol. 14, 406-413

\title{
The Action of Isonicotinic Acid Hydrazide on the Metabolism of Mycobacterium smegmatis
}

\author{
By PAULINE MEADOW* \\ Department of Bacteriology, Guy's Hospital Medical School, London, S.E. 1
}

SUMMARY: The effect of various substrates on the oxygen uptake of Mycobacterium smegmatis grown in the presence of glucose and glycerol was investigated; isoniazid had no effect on these oxidations. Three different effects of isoniazid on the metabolism of this organism were demonstrated. (i) Growth of the organism in a minimal medium in the Warburg apparatus was prevented by the addition of isoniazid, the amount required being related to the number of organisms present, their age and their sensitivity to the drug as measured by serial dilution in the test tube. (ii) In confirmation of the report of Zeller, Barsky, Berman \& Fouts (1952) it was found that isoniazid inhibited the oxidation of putrescine by $\boldsymbol{M}$. smegmatis. (iii) Following the work of Gray (1953) the effect of isoniazid on the oxidation of various substrates by $M$. smegmatis grown in the absence of glucose and glycerol was studied. Isoniazid caused a $30 \%$ inhibition of the oxidation of acetate. The amount of isoniazid required to inhibit the oxidation of putrescine and acetate was the same whether the strain used was sensitive or resistant to isoniazid as measured by growth in the test tube.

In an attempt to discover the mechanism by which isonicotinic acid hydrazide (isoniazid) inhibits the growth of mycobacteria, the effect of isoniazid on their respiration was investigated. Preliminary work involved a study of the oxidative metabolism of the saprophytic organism Mycobacterium smegmatis.

\section{METHODS}

Organisms. The parent strain of Mycobacterium smegmatis was obtained from the National Collection of Type Cultures (7011). It was maintained on nutrient agar and subcultivated into Dubos fluid medium $48 \mathrm{hr}$. before use. This parent strain, $M$. smegmatis $\mathrm{S}$, was inhibited by $8 \mu \mathrm{g}$. isoniazid $/ \mathrm{ml}$. By serial transfer from the highest concentration of isoniazid in which growth occurred, into higher concentrations of the drug, strains were obtained which were resistant to $200 \mu \mathrm{g}$. isoniazid $/ \mathrm{ml}$. (M. smegmatis $\mathrm{R}_{1}$ ) and to $400 \mu \mathrm{g}$. isoniazid/ml. (M. smegmatis $\mathbf{R}_{2}$ ).

\section{Media}

Dubos fluid medium (Dubos \& Davis, 1946). This was supplied by the Southern Group Laboratory, Park Hospital, Hither Green, London, S.E. 13. The basal medium containing Tween 80 was supplied in $100 \mathrm{ml}$. amounts, and a $9 \%(\mathrm{w} / \mathrm{v})$ solution of bovine albumin fraction V (Armour) in water in $5 \mathrm{ml}$. amounts. The bovine albumin from several bottles was pooled, and resterilized by filtration through a Seitz E.K. filter. For use, $4 \mathrm{ml}$. of this albumin solution was added to each $100 \mathrm{ml}$. of Dubos medium.

* Present address: Department of Chemical Pathology, University College Hospital Medical School, London, W.C. 1. 
Dubos \& Middlebrook medium for heavy yield of organisms (Dubos \& Middlebrook, 1947). This was prepared by adding magnesium sulphate, sodium citrate, glycerol, and glucose to the Dubos medium as used above.

Fumarate medium. This was a modification of the Dubos medium, and contained, in $1 \mathrm{l}$. of medium: $\mathrm{KH}_{2} \mathrm{PO}_{4}, 1 \mathrm{~g}$; $\mathrm{Na}_{2} \mathrm{HPO}_{4} .12 \mathrm{H}_{2} \mathrm{O}, 6 \cdot 5$ g.; $\mathrm{MgSO}_{4} .7 \mathrm{H}_{2} \mathrm{O}, 0 \cdot 4 \mathrm{~g}$; casein hydrolysate $(20 \%$, w/v), $10 \mathrm{ml}$; Tween 80 , $10 \%(\mathrm{v} / \mathrm{v}), 5 \mathrm{ml}$; sodium fumarate $1 \cdot 7 \mathrm{~g}$. The $\mathrm{pH}$ value was adjusted to $6 \cdot 8$ and the medium sterilized by autoclaving in $100 \mathrm{ml}$. amounts at $10 \mathrm{lb}$. for $10 \mathrm{~min}$.

\section{Sensitivity tests}

These were carried out in test tubes $\left(6 \times \frac{5}{8} \mathrm{in}\right.$.), each containing $4 \mathrm{ml}$. of Dubos fluid medium. The inoculum was 1 drop $(0.02 \mathrm{ml}$.) of a $48 \mathrm{hr}$. culture in Dubos medium. Incubation was at $37^{\circ}$.

\section{Warburg experiments}

The organisms were grown in Roux bottles, each containing $100 \mathrm{ml}$. of medium, and incubated flat for 3 days before harvesting. The cultures were collected by centrifugation at 3000 r.p.m. for 15 min., washed by resuspending in $0.2 \mathrm{M}$-phosphate buffer $(\mathrm{pH} 6 \cdot 8)$ and centrifuged again at 3000 r.p.m. for a further $15 \mathrm{~min}$. For use the organisms were resuspended in phosphate buffer, and the suspensions standardized in the Unicam absorptiometer. One ml. of this suspension was used in each Warburg vessel which had a total fluid volume of $3.2 \mathrm{ml}$. All substances to be tested were dissolved so that the desired amount was contained in $\mathbf{0 . 6} \mathrm{ml}$. distilled water and added from the side arm; $0.2 \mathrm{ml} .20 \% \mathrm{KOH}$ was used to absorb $\mathrm{CO}_{2}$. The temperature of the bath was $37^{\circ}$ and the shaking rate 100 strokes/min. Results are expressed in terms of $Q_{\mathrm{O}_{2}}$, i.e. $\mu$ l. $\mathrm{O}_{2}$ taken up/mg. dry wt. organisms $/ \mathrm{hr}$.

\section{RESULTS}

\section{Oxidative reactions of organisms grown in Dubos \& Middlebrook medium}

In order to obtain a high yield of organisms and so counteract some of the experimental difficulties of using mycobacteria, the organisms were grown in Dubos \& Middlebrook medium. This contains salts, glycerol, casein hydrolysate and glucose, and a good yield was obtained after 3 days of incubation.

Effect of isoniazid on the oxidation of single substrates. The effect of various single substrates upon the oxygen uptake of washed cell suspensions of Mycobacterium smegmatis was investigated. The substances tested included glycerol, glucose, components of the Krebs citric acid cycle, several amino acids, and certain benzoic acid derivatives. The results obtained and the effect of isoniazid on these oxidations are shown in Table 1. Although the concentration of isoniazid used $\left(10^{-1} \mathrm{M}\right)$ was far greater than that required to inhibit growth in the test tube $\left(5 \times 10^{-4} \mathrm{M}\right)$, the addition of isoniazid caused no change in the $Q_{\mathrm{O}_{2}}$ under the conditions used, in the presence of any of the substrates tested. 
In view of the work of Umbreit (1953) on the effect of streptomycin on the reaction between pyruvate and oxaloacetate, the effect of isoniazid on oxygen uptake from oxaloacetate in the presence of pyruvate was tested. This also was unchanged by the addition of isoniazid.

Table 1. Effect of isoniazid on oxygen uptake of Mycobacterium smegmatis

Substrate (conc. $\mathbf{1 0}^{-\mathbf{2}} \mathrm{M}$ )
No addition
Asparagine
Sodium acetate
$\alpha$-Alanine
$\beta$-Alanine
Sodium benzoate
-Aminobenzoic acid
-Hydroxybenzoic acid
Anthranilic acid
Glutamic acid
Glyceraldehyde
Glycerol
Glucose
L-Histidine HCl
Sodium lactate
Sodium citrate
L-Leucine
Sodium oxaloacetate
Sodium pyruvate
Serine
Sodium succinate
Tyrosine
Tryptophan
Oxaloacetate + pyruvate

$\begin{array}{lc}Q_{\mathrm{O}_{2}} & \begin{array}{c}Q_{\mathrm{O}_{2}} \text { in presence of } \\ (0 \cdot 1 \mathrm{M}) \text { isoniazid }\end{array} \\ 13 \cdot 6 & 13 \cdot 6 \\ 39 \cdot 4 & 39 \cdot 2 \\ 49 \cdot 8 & 49 \cdot 8 \\ 38 \cdot 6 & 38 \cdot 6 \\ 40 & 40 \cdot 5 \\ 37 \cdot 8 & 38 \cdot 1 \\ 13 \cdot 0 & 13 \cdot 2 \\ 13 \cdot 5 & 13 \cdot 8 \\ 12 \cdot 9 & 12 \cdot 8 \\ 28 \cdot 2 & 28 \cdot 0 \\ 26 \cdot 8 & 26 \cdot 8 \\ 22 \cdot 4 & 22 \cdot 5 \\ 29 \cdot 6 & 30 \cdot 1 \\ 26 \cdot 3 & 27 \\ 29 \cdot 4 & 30 \cdot 2 \\ 13 \cdot 6 & 13 \cdot 2 \\ 29 \cdot 1 & 28 \cdot 5 \\ 26 \cdot 4 & 27 \cdot 1 \\ 34 & 33 \cdot 4 \\ 30 & 30 \\ 31 & 29 \cdot 6 \\ 20 \cdot 4 & 20 \\ 29 \cdot 4 & 29 \cdot 2 \\ 60 \cdot 8 & 60 \cdot 3\end{array}$

Effect of isoniazid on the oxygen uptake of 'growing' organisms. Since no inhibition of oxidative reactions by isoniazid was found with resting suspensions of Mycobacterium smegmatis, it was decided to study the effect of isoniazid on oxygen uptake during growth in the Warburg apparatus. A suspension of M. smegmatis already growing at $37^{\circ}$ in Dubos \& Middlebrook medium was transferred directly, without washing, to Warburg flasks. In the presence of isoniazid $\left(10^{-4} \mathrm{M}\right)$ the $Q_{\mathrm{O}_{2}}$ was steady over a period of $7 \mathrm{hr}$., but in its absence the $Q_{\mathrm{O}_{2}}$ increased at a constant rate (Fig. 1). That this increase in the rate of oxygen uptake in the absence of isoniazid was due to growth, is suggested by the following points:

(1) At the end of $7 \mathrm{hr}$. there was an increase in the mass of organisms. This was visible to the naked eye and was also shown by measuring the turbidity in the Unicam absorptiometer.

(2) By plotting the oxygen uptake against the square of time, a linear relation was shown (Fig. 1). This arithmetic type of growth pattern was found by Fisher \& Kirchheimer (1952) to be typical of the growth of mycobacteria under certain conditions. Later Halpern \& Kirchheimer (1954) showed that the growth reverted to the more usual logarithmic type of growth on shaking. 
(3) Organisms grown for $24 \mathrm{hr}$. before transferring to Warburg flasks were more active than those grown for $48 \mathrm{hr}$. or longer.

(4) More dilute suspensions of organisms showed a more rapid increase in rate of oxygen uptake than heavier ones.

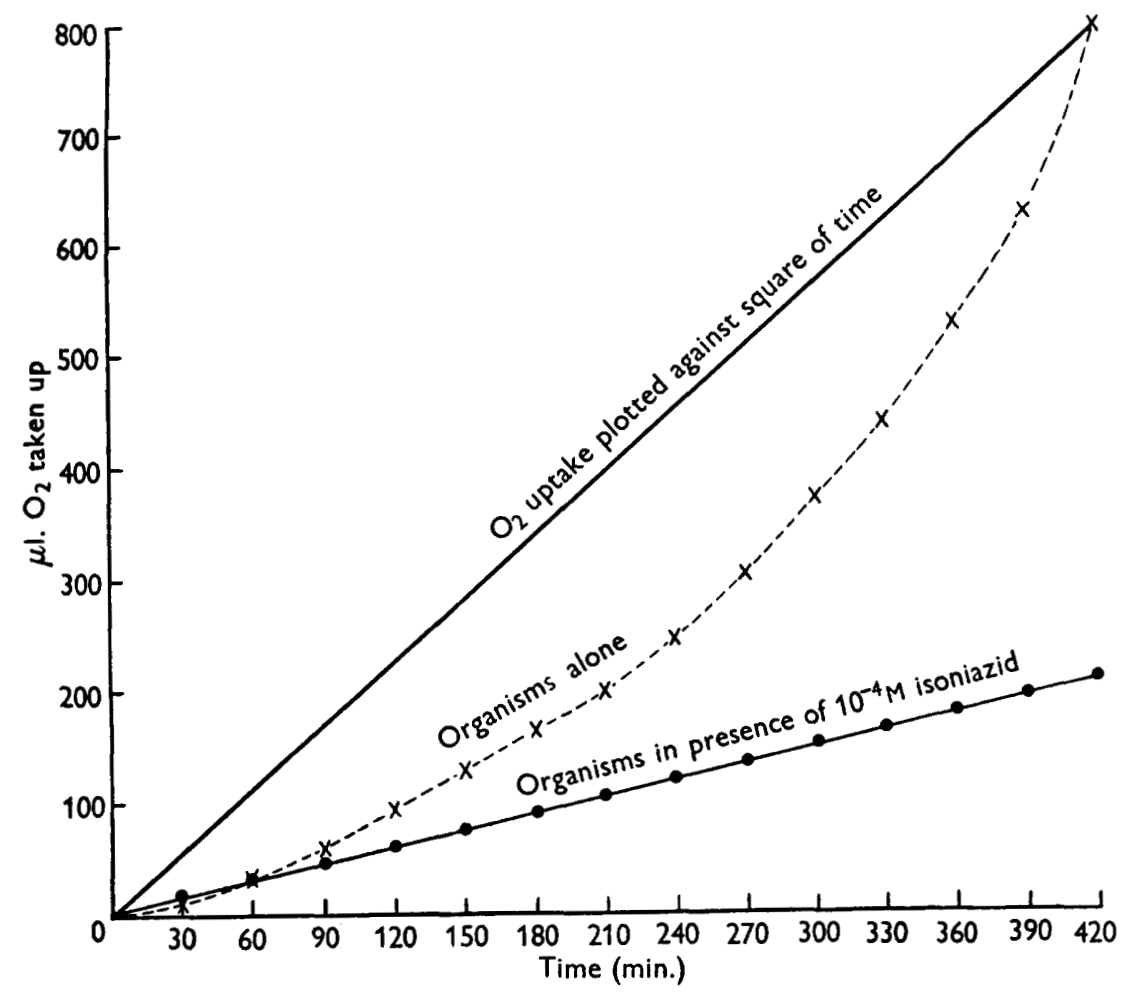

Fig. 1. Growth of Mycobacterium smegmatis in the Warburg apparatus and its inhibition by isoniazid.

Attempts were made to simplify this system which showed isoniazid inhibition. An increase in $Q_{\mathrm{O}_{2}}$ was also obtained after washing the organisms twice with phosphate buffer, and resuspending them in $0 \cdot 2 \mathrm{M}$-phosphate ( $\mathrm{pH} \mathrm{6 \cdot 8)}$ containing $0.2 \%(\mathrm{w} / \mathrm{v})$ casein hydrolysate and $0 \cdot 1 \mathrm{M}$-glycerol. The rate of increase of $\mathrm{QO}_{2}$ in this simplified system was unaffected by the addition of albumin, Tween 80, magnesium sulphate, sodium citrate, glucose, tuberculin purified protein derivative (P.P.D.), phthiocol, $p$-aminobenzoic acid or pyridoxin.

When the casein hydrolysate in the simplified system was replaced by putrescine, aspartic acid, asparagine, serine, ammonium sulphate or ammonium citrate the 'growth' effect was not observed. The glycerol could not be replaced by glyceraldehyde or glucose. The concentration of isoniazid required to inhibit 'growth' was related to that required to inhibit growth in Dubos fluid medium in the test tube (Table 2). 
Antagonists. Several substances were tested as antagonists of isoniazid inhibition in this system. It was thought possible that inhibition of growth by isoniazid might be due to its competitive antagonism with pyridoxin or nicotinic acid, because of similarity of structure. Pyridoxin $\left(10^{-2}\right.$ to $\left.10^{-5} \mathrm{M}\right)$, pyridoxal $\left(5 \times 10^{-5}\right.$ to $\left.10^{-7} \mathrm{M}\right)$ and nicotinic acid $\left(10^{-2}\right.$ to $\left.10^{-5} \mathrm{M}\right)$ had no effect on the system; they did not change the rate of increase of oxygen uptake nor

Table 2. Relation between amounts of isoniazid required to inhibit growth of strains of Mycobacterium smegmatis in test tubes and in Warburg flasks

$\begin{array}{lcc}\text { Organism } & \overbrace{\begin{array}{c}\text { In test tube } \\ (\mu \mathrm{g} . / \mathrm{ml} .)\end{array}}^{\text {Isoniazid required for inhibition }} & \begin{array}{c}\text { In Warburg } \\ \text { flasks } \\ (\mu \mathrm{g} . / \mathrm{ml} .)\end{array} \\ M . \text { smegmatis } \mathbf{S} & 6 & 10 \\ M . \text { smegmatis } \mathbf{R}_{1} & 200 & 200 \\ M . \text { smegmatis } \mathbf{R}_{2} & 400 & 420\end{array}$

Table 3. Effect of isoniazid on acetate oxidation by Mycobacterium smegmatis grown in the absence of glucose or glycerol

\begin{tabular}{|c|c|c|c|c|}
\hline Strain & Isoniazid (M) & $Q_{0_{2}}$ (basal) & $\begin{array}{c}Q_{\mathrm{O}_{2}} \text { in presence } \\
\text { of acetate } \\
(0 \cdot 1 \mathrm{M})\end{array}$ & $\begin{array}{c}\text { Inhibition } \\
(\%)\end{array}$ \\
\hline M. smegmatis $\mathrm{S}$ & $\begin{array}{c}0 \\
10^{-2} \mathrm{Mr} \\
10^{-3} \mathrm{M}\end{array}$ & $\begin{array}{l}1 \cdot 3 \\
1 \cdot 3 \\
1 \cdot 3\end{array}$ & $\begin{array}{c}13 \\
9 \cdot 5 \\
13 \cdot 1\end{array}$ & $\overline{31}$ \\
\hline M. smegmatis $\mathrm{R}_{1}$ & $\begin{array}{c}0 \\
10^{-2} M \\
10^{-3} M\end{array}$ & $\begin{array}{l}1 \cdot 32 \\
1 \cdot 31 \\
1 \cdot 33\end{array}$ & $\begin{array}{r}13 \cdot 0 \\
9 \cdot 4 \\
13 \cdot 0\end{array}$ & $\begin{array}{r}\overline{31} \\
0\end{array}$ \\
\hline
\end{tabular}

the action of isoniazid in suppressing this. Manganese chloride $\left(10^{-3}\right.$ to $\left.10^{-4} \mathrm{M}\right)$, sodium citrate $\left(10^{-2}\right.$ to $\left.10^{-4} \mathrm{M}\right)$ and haemin $\left(10^{-3}\right.$ to $\left.10^{-6} \mathrm{M}\right)$ were used, following reports by Fisher (1954) that they antagonized the inhibition by isoniazid of growth of Mycobacterium tuberculosis $\mathbf{H 3 7} \mathbf{R v}$. The screening plate technique used as described by King, Knox \& Woodroffe (1953), indicated that salicylic acid hydrazide, nicotinic acid hydrazide and benzhydrazide, antagonized the inhibitory action of isoniazid on $M$. smegmatis in growth (Anderson, King, Knox \& Meadow, 1953). These substances were also tested at concentrations of $10^{-2}$ to $10^{-4} \mathrm{M}$ in the above system but were without effect.

\section{Oxidative reactions of cells grown in the absence of glucose and glycerol}

Gray (1953) showed that inhibition by isoniazid of oxygen uptake by mycobacteria occurred only when the organisms were grown in a medium free from glucose and glycerol, or were starved in aerated phosphate buffer after growth. The effect of isoniazid on the oxidation of single substrates by Mycobacterium smegmatis grown in the absence of glucose and glycerol was therefore tested. The organisms were grown for 3 days in the fumarate medium; they had a much lower basal $Q_{\mathrm{O}_{2}}$ than the organisms used previously. Table 3 shows the 
results obtained when isoniazid-sensitive and isoniazid-resistant strains of M. smegmatis were used with acetate as substrate. $10^{-2} \mathrm{M}$-isoniazid caused a $30 \%$ inhibition of the oxygen uptake from acetate whether or not the strain used was sensitive to isoniazid as measured by growth in Dubos medium in the test tube.

\section{Diamine oxidase}

One of the earlier reports of enzyme inhibition by isoniazid was that of Zeller et al. (1952) who described the effect of isoniazid on the diamine oxidase of cell suspensions and soluble enzyme preparations of Mycobacterium smegmatis. The most active of the diamine oxidases in this organism is the putrescine

Table 4. Action of isoniazid on putrescine oxidase of Mycobacterium smegmatis

The organisms were grown in Dubos \& Middlebrook medium containing $0.5 \%(w / v)$ putrescine.

$\begin{array}{cccc}\begin{array}{c}\text { Putrescine } \\ \text { concentration }\end{array} & \begin{array}{c}\text { Isoniazid } \\ \text { concentration }\end{array} & Q_{\mathrm{O}_{2}} & \begin{array}{c}\text { Inhibition } \\ (\mathrm{M})\end{array} \\ (\mathrm{M}) & & (\%) \\ 0 & 0 & 4 \cdot 7 & - \\ 0 \cdot 01 & 0 & 12 \cdot 1 & - \\ 0 \cdot 01 & 10^{-2} & 6 \cdot 5 & 72 \\ 0 \cdot 01 & 10^{-3} & 6 \cdot 45 & 72 \\ 0 \cdot 01 & 10^{-4} & 12 \cdot 0 & 0\end{array}$

oxidase, and this was tested in the Warburg apparatus and the effect of adding isoniazid studied. In confirmation of the report of Zeller et al. (1952) it was found that putrescine was oxidized by washed suspensions of $M$. smegmatis and that isoniazid at $10^{-3} \mathrm{M}$ caused a $72 \%$ inhibition of this oxidation in both sensitive and resistant strains (Table 4).

\section{Arginine decarboxylase}

Yoneda \& Asano (1953) reported that the arginine decarboxylase of a strain of Escherichia coli was inhibited by high concentrations of isoniazid, the inhibition being reversed by pyridoxin and more effectively by high concentrations of pyridoxal. This work was repeated with Escherichia coli communius (NCTC 6064) grown for $16 \mathrm{hr}$. at $25^{\circ}$ in $2 \%(\mathrm{w} / \mathrm{v})$ glucose broth to which had been added $0.001 \%(\mathrm{w} / \mathrm{v})$ arginine. The arginine decarboxylase was measured by the evolution of carbon dioxide after the addition of arginine (to $3 \times 10^{-3} \mathrm{M}$ ) to washed suspensions in McIlvaine's citric acid buffer ( $\mathrm{pH} 4 \cdot 2)$. As shown in Table 5, isoniazid inhibited the action of the arginine decarboxylase, and this inhibition was partially reversed by pyridoxin $\left(10^{-2} \mathrm{M}\right)$. Attempts were made to demonstrate a similar effect with washed suspensions of Mycobacterium smegmatis. The organisms were grown in broth containing $2 \%(\mathrm{w} / \mathrm{v})$ glucose and $0.001 \%(\mathrm{w} / \mathrm{v})$ arginine at 25 and at $37^{\circ}$, and also in Dubos \& Middlebrook medium to which $0.001 \%(\mathrm{w} / \mathrm{v})$ arginine had been added. No arginine decarboxylase was found in any of these organisms. 
Table 5. The effect of isoniazid and pyridoxin on the arginine decarboxylase of Escherichia coli communius

Isoniazid
concentration
$(\mathrm{M})$
0
$10^{-2}$
$10^{-3}$
$10^{-4}$
0
$10^{-2}$

Pyridoxin
concentrati
(घ)
0
0
0
0
$10^{-2}$
$10^{-2}$

$Q_{0_{2}}$

60
7
48
68
62
24

\begin{tabular}{c} 
Inhibition \\
$(\%)$ \\
- \\
88 \\
20 \\
0 \\
0 \\
\hline
\end{tabular}

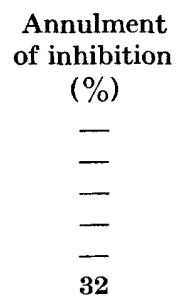

\section{DISCUSSION}

The systems so far shown to be inhibited by isoniazid are the putrescine oxidase and the acetate oxidase of Mycobacterium smegmatis, 'growth' of $\boldsymbol{M}$. smegmatis in a minimal medium and the arginine decarboxylase of Escherichia coli. It appears unlikely that inhibition of the putrescine oxidase of Mycobacterium smegmatis is the only, or indeed the major, site of action of isoniazid for two main reasons: (1) Inhibition of diamine oxidases is a general reaction given by many substances (including hydroxylamine, nicotinic acid hydrazide and streptomycin), and it does not appear to be related to their antituberculous activity (Zeller, Owen \& Karlson, 1951). (2) Certain diamines, notably spermine, are inhibitory to the growth of tubercle bacilli only in the presence of the diamine oxidase (Hirsch \& Dubos, 1952). If isoniazid acts only as an inhibitor of the diamine oxidase, it should therefore permit growth, rather than inhibit it, in the presence of diamines. No such isoniazid dependence has been demonstrable in growth experiments in the presence of diamines. The presence of an arginine decarboxylase in $\boldsymbol{M}$. smegmatis has not been demonstrated. It seems unlikely, therefore, that inhibition of arginine decarboxylase by isoniazid plays a part in its inhibition of the growth of mycobacteria.

The chief effect of isoniazid on 'growth' as followed in the Warburg apparatus seems to resemble fairly closely conditions in the test tube, in which growth inhibition is known to occur, since the amounts of isoniazid required for inhibition in the two systems are comparable. This is not, however, an easy technique with which to work since the low concentrations of organisms required for optimum 'growth' necessitate the use of organisms which are not clumped together. Mechanical grinding, however gentle, prevents the 'growth' effect, and it is therefore difficult to be sure of obtaining reasonably uniform distributions of the organisms into each Warburg flask. It was thought probably not profitable to continue using the technique as a means of demonstrating the activity of isoniazid, particularly in view of the fact that no such effect was demonstrable with Mycobacterium tuberculosis var. bovis (B.C.G.).

The most hopeful line of approach to the problem appears to be the inhibition of acetate oxidation by isoniazid, using organisms grown in a medium free from glucose and glycerol. However, the use of Mycobacterium smegmatis in 


\section{Action of isoniazid on Mycobacterium smegmatis}

this work is limited by the fact that its sensitivity to isoniazid as measured by this technique is not related to its sensitivity to the drug in growth tests, and that it is considerably less sensitive to isoniazid than other members of the genus Mycobacterium such as $M$.tuberculosis var. hominis (H37 Rv and $\mathrm{H} 37 \mathrm{Ra})$ and M. tuberculosis var. bovis (B.C.G.).

I wish to thank the Guy's Hospital Endowments Fund for a maintenance grant.

\section{REFERENCES}

Anderson, K. F., King, M. B., Knox, R. \& Meadow, P. (1953). The in vitro action of isoniazid. Proc. VI Congr. int. Microbiol. 1, 139.

Dubos, R. J. \& Davis, B. D. (1946). Factors affecting the growth of tubercle bacilli in liquid media. J. exp. Med. 83, 409.

Dubos, R. J. \& Middlebrook, G. (1947). Media for tubercle bacilli. Amer. Rev. Tuberc. 56, 334.

Fisher, M. W. (1954). The antagonism of the tuberculostatic action of isoniazid by hemin. Amer. Rev. Tuberc. 69, 469.

Fisher, M. W. \& Kirchueimer, W. F. (1952). Studies on the growth of mycobacteria. I. The occurrence of arithmetic linear growth. Amer. Rev. Tuberc. 66, 758.

Gray, C. T. (1953). Nature of the action of isonicotinic acid hydrazide on the mycobacteria. Proc. VI Congr. int. Microbiol. 1, 179.

Halpern, B. \& Kirchheimer, W. F. (1954). Studies on the growth of mycobacteria. II. The effect of oxygenation and aeration on the growth pattern of mycobacteria. Amer. Rev. Tuberc. 70, 665.

Hirsch, J. G. \& Dubos, R. J. (1952). The effect of spermine on tubercle bacilli. J. exp. Med. 95, 191.

King, M. B., Knox, R. \& Woodrofre, R. C. (1953). Investigation of antitubereulous substances. An agar diffusion method using Mycobacterium smegmatis. Lancet, 264, 573.

Umbreit, W. W. (1953). The action of streptomycin. VI. A new metabolic intermediate. J. Bact. 66, 74 .

Yoneda, M. \& Asano, N. (1953). Competitive action of isonicotinic acid hydrazide and pyridoxal in the amino acid decarboxylation of Escherichia coli. Science, $117,277$.

Zeller, E. A., Barsky, J., Berman, E. R. \& Fouts, J. R. (1952). Action of isonicotinic acid hydrazide and related compounds on enzymes involved in the autonomic nervous system. J. Pharmacol. 106, 427.

Zeller, E. A., Owen, C. A. Jun. \& Karlson, A. G. (1951). Diamine oxidase of Mycobacterium smegmatis: effect of streptomycin and dihydrostreptomycin. J. biol. Chem. 188, 623. 